\title{
VISUAL SALIENCY BASED BRIGHT LESION DETECTION AND DISCRIMINATION IN RETINAL IMAGES
}

\author{
Ujjwal, K Sai Deepak, Arunava Chakravarty, Jayanthi Sivaswamy \\ Center for Visual Information Technology \\ International Institute of Information Technology \\ Gachibowli, Hyderabad, India
}

\begin{abstract}
Abnormality detection is the first step performed by doctors during evaluation of medical images in image based diagnosis, followed by disease-specific evaluation of abnormalities. Perception studies have shown that experts primarily focus on abnormal structures during visual examination for diagnosis. One way to model this behavior in automated image analysis is through visual saliency computation. In this paper, we investigate the potential role of visual saliency for computer aided diagnosis algorithm design. We propose a framework for detecting abnormalities that uses visual saliency computation for sparse representation of the image data that preserves the essential features of a normal image. The proposed method is evaluated for the task of bright lesion detection and classification in color retinal images which is of significance in disease screening. An evaluation of the proposed approach on 5 publicly available datasets yielded area under ROC curve of 0.88 to 0.98 for the detection task and accuracies ranging from 0.93 to 0.96 for lesion discrimination. These results establish visual saliency as an alternate avenue for automated abnormality detection.
\end{abstract}

Index Terms - Abnormality Detection, Bright Lesion Detection, Color Fundus Images, Visual Saliency

\section{INTRODUCTION}

Abnormality detection from medical images has a fundamental role in medical diagnosis. It is of importance in the context of life and sight threatening diseases where early detection of abnormalities using computer aided diagnosis (CAD) can help in planning an effective disease management program for the patient. Improving CAD systems is an active area of research. Medical image perception has been actively studied since the eighties not only to model visual search strategies in experts but also to help improve detection by experts. For example, [1] collected eye/gaze tracking data of radiologists as they viewed chest X-ray images to derive a model which explains the sequence of events from the time of viewing an image to diagnostic decision making on presence of tumors by experts. The foveation points in a gaze map is generally treated as signaling relevance of the image regions based on conspicuity and/or clinical significance. Recently, [2] extended the perception study to brain CT images for lesion detection. Here, gaze data from experts and other control subjects were analyzed and compared with saliency maps derived from the same images [3] which locates conspicuous image areas. It was found that the saliency maps had a good correlation with gaze maps, though experts also tended to examine clinically relevant areas which were less conspicuous (low saliency). This indicates the role of top down or domain knowledge in visual examination. These findings have also been confirmed in our study on Chest X-ray and color retinal images [4].

In this paper, we propose a visual saliency based framework for abnormality detection. Bright lesions on the retinal surface such as Hard Exudates (HE) and drusen are important signs of sight-threatening diseases like diabetic retinopathy (DR) and age-related macular degeneration (AMD) respectively. We describe how this framework can be applied to the problem of bright lesion detection and classification in retinal images.

\section{RELATED WORK}

Currently, the predominant strategy for bright lesion detection is to detect/segment candidates and classify them as lesions/non-lesions. [5] adopts this and demonstrates it on a mixed dataset of images with HE and drusen. Two recent methods for bright lesion detection that use global approach include one which constructs a dictionary using SURF features [6] and ours where a subspace of normal images is learnt using motion patterns [7]. For HE detection, Haar wavelet features have been used to assign confidence values to candidate lesions obtained through global thresholding [8] and AM-FM features have also been used for image level classification [9] derives. Drusen detection has been attempted with wavelet-based [10] and PCA based [11]filtering at a global level. In addition to global approach to HE or drusen detection receiving less attention, there has also been scant attention paid to devising global methods for detection of both these lesions. 


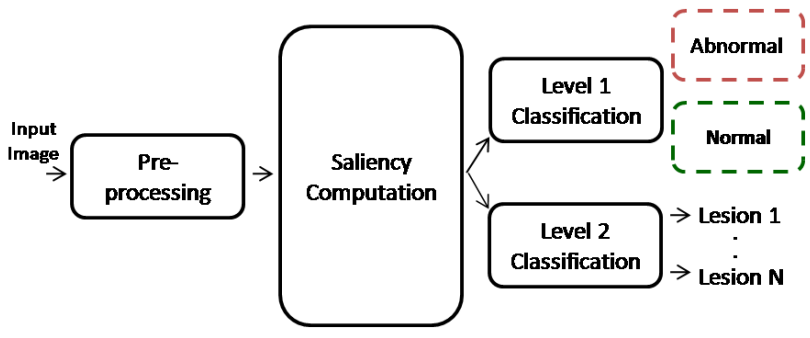

Fig. 1. Block diagram for proposed saliency based framework

\section{PROPOSED METHOD}

We propose a novel and global approach for abnormality detection based on visual saliency. Saliency is a biologically motivated concept describing the tendency of a region of an image to attract an observer's attention - a greater tendency meaning greater saliency.In medical images, domain knowledge about disease, anatomy etc., also plays a role in determining the importance of a region [2][4]. Computational modeling of saliency is an active area of research. The aim is to create a saliency map for a given image indicating the saliency strength at every pixel. We propose a system for saliency-based bright lesion detection consisting of 3 stages: i) preprocessing to boost local contrast of lesions to handle variations in brightness; ii) saliency computation to obtain a sparse representation for the image; iii) feature extraction and classification which can be performed in two levels using saliency map. An image can be classified as normal or having bright lesions (level 1) or an abnormal image can be identified as belonging to HE or drusen class (level 2). Figure 1 shows the schematic of the system.

\subsection{Pre-processing}

Variabilities in bright lesions in their shapes, sizes and brightness are handled in pre-processing. The green channel of the given image $I$ is top-hat filtered using a disk-shaped structuring element to boost the local contrast to obtain $I_{t}$. Next, to minimize the effect of uneven illumination, the background illumination is estimated (using median filtering) and subtracted from $I_{t}$ to generate $I_{t s}$. Finally, a contrast stretching step is done as follows: $I_{p}=(\alpha)^{I_{t s}}$, where $\alpha$ is found empirically. $I_{p}$ is the input for saliency computation.

\subsection{Saliency computation}

The Spectral residual (SR) [12] model which aims at detecting proto-objects from images was chosen for saliency computation based on our experiments reported in [4]. This model is based on the assertion that for a given image $f$ saliency is signaled by deviations from the envelope of the log spectrum of $f$. We denote the Fourier spectrum of $f$ as $\mathcal{F}(f)=$ $\mathcal{M}$.exp $(j(\mathcal{P}))$. The saliency map $\mathcal{S}$ is found by the following sequence of computations :

$\mathcal{R}=\log (\mathcal{M})-h_{n} * \log (\mathcal{M}) ; \mathcal{S}=g * \mid \mathcal{F}^{-1}\left[\left.\exp (\mathcal{R}+j \mathcal{P}]\right|^{2}\right.$ where, '*' denotes convolution, $h_{n}$ and $g$ are smoothing filters. All computations are done after downsampling the given image $f$ to $64 \times 64$ and interpolating the result back to original size. This leads to a diffused appearance of proto-objects in the saliency map. We addressed this in [4] by performing saliency computation on a pyramidal representation of $f$ and then combining the results. The final saliency map is obtained by interpolating the saliency maps at each level of the pyramid back to the original image size and adding them (with normalization). Two sample images along with their computed saliency maps are shown in Figure 2. It is notable that the maps are sparse and the main proto-objects (subtle as well as prominent lesions and optic disc) in the input image are captured and localized.

\subsection{Level 1 classification}

The saliency map $\mathcal{S}$ available from the previous stage captures the optic disk (a bright anatomical structure) and some of the bright reflex along the major vessel. These distractors can be much larger and brighter than the subtle and small bright lesions. Hence, we use the Generalized Motion Patterns (GMP) we have proposed earlier [7] to derive a robust representation of $\mathcal{S}$. GMP is obtained as follows. Since retina is a circular structure, whereas the given image is rectangular, a circular region of interest (ROI) is first extracted around the image center. A rotational motion is applied to the ROI in steps, with the image center as the pivot, to generate a set of rotated images. These are coalesced using a maximum operation. Further details can be found in [7]. The rotational motion and maximum-based combination serve not only to enhance any locally bright structure by spatially extending them across the image, but also average out the normal variabilities that have low saliency values in the saliency map. The end result of this step is the GMP $M$ and the ToP (Texture of Projections) descriptor [13], which is based on computing the texture (quantified by LBP i.e. Local Binary Patterns) of projections, is derived for $M$. In the present work, the motivation is to learn the class of normal images so that any significant deviation from it is marked as an abnormal image. We implement this learning using a K-Nearest neighbor data description (K-NN DD) classifier, which is known to perform very well when the features distinguishing the target class are sufficiently distinct.The ToP of GMP is such a distinct feature. A KNN-DD is trained on the ToP features extracted from a set of normal retinal images. Given a test image, it is said to be normal if the number of its nearest neighbors within a specified (Euclidean) distance $d$ is greater than a certain threshold value which is empirically determined.

\subsection{Level 2 classification}

A saliency map provides very useful source of information on locations of abnormalities in an image and thus it should also help in discriminating between images with HE and drusen. We used a simple method to confirm this hypothesis. The given retinal image was divided into a fixed number of patches. Patches with lesions $\left(P_{L}\right)$ were identified as patches with saliency values greater than $x \%$ of the highest saliency 
Table 1. Distribution of images used from public datasets

\begin{tabular}{lcc}
\hline \hline Dataset & Normal cases & Abnormal cases \\
\hline \hline Hard Exudates & & \\
\hline HEI-MED[8] & 68 & 54 \\
\hline DiaretDB[14] & 27 & 38 \\
\hline MESSIDOR[7] & 275 & 125 \\
\hline \hline Drusen & & \\
\hline ARIA[15] & - & 92 \\
\hline STARE[16] & - & 79 \\
\hline \hline Combined & 370 & 388 \\
\hline \hline
\end{tabular}

value. Local binary patterns (LBP) features were extracted from the image (the green plane) for every $P_{L}$ and used to classify it as having $\mathrm{HE}$ or drusen using an SVM classifier with RBF kernel. The final, image-level decision was based on majority voting among all $P_{L}$.

\section{EXPERIMENTS AND RESULTS}

Experiments for bright lesion detection (level 1) and discrimination (level 2) were performed on 5 publicly available datasets. Sensitivity and specificity are measured for the detection task. Validation was also performed on a combined dataset to assess the robustness of the proposed framework.

\subsection{Datasets}

Five public datasets, listed in table 1, were used in our experiments. HEI-MED, Diaretdb0,1 (combined and named as DiaretDB) and MESSIDOR are used for HE detection whereas ARIA and STARE are used for drusen detection. Normal cases from drusen datasets are ignored due to their poor resolution and imaging artifacts. Instead normal images from HEI-MED were used for evaluation. A superset (Combined) was created by combining all datasets.

\subsection{Results}

Pre-processing was performed on retinal images with $\alpha=$ $\frac{\exp (1)}{2}$. Saliency map computation was done on image pyramid with 5 levels. Sample images and saliency maps can be seen in figure 2. Figure 3 shows sub-images with low contrast, bright lesions and corresponding saliency maps capturing these lesions well.

Bright Lesion Detection (level 1)- Feature extraction was performed on $\mathcal{S}$ using GMP generated with parameters as in [7]. The ToP descriptor was derived taking Radon transform with orientations between $0^{\circ}-180^{\circ}$ with a step size of $3^{\circ}$ and 256 bins.

At level 1, images were classified into normal/abnormal classes using the KNN-DD classifier with $K=3$. The number of normal cases used for training the classifier were 30, 12, 125 and 167 for HEI-MED, DiaretDB, MESSIDOR and Combined datasets, respectively. The computed sensitivity (SN) and specificity (SP) for lesion detection are presented in Table 2. Receiver operating characteristic (ROC) curve for L1 is shown in figure 4. A 5-fold cross validation was used to ensure that there is no bias due to the selection of training and testing set.

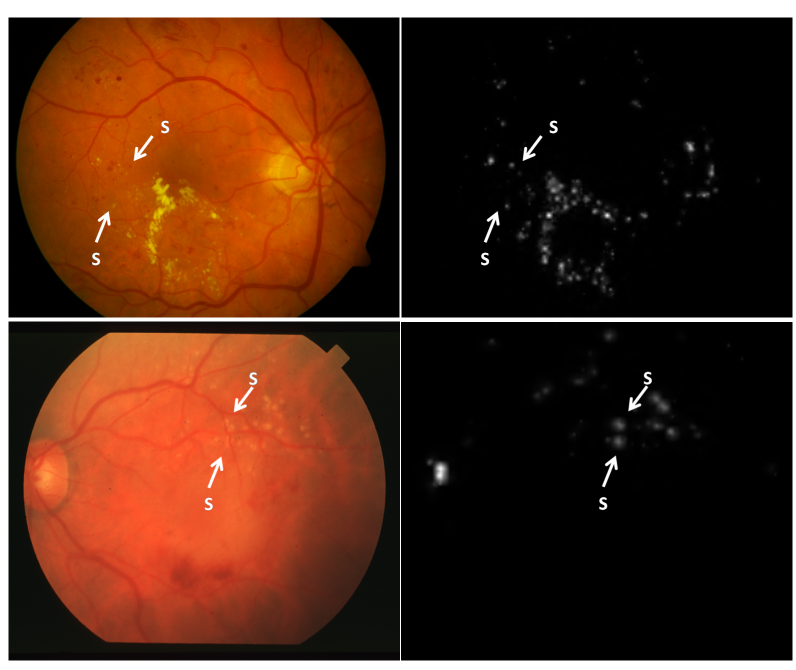

Fig. 2. Retinal images (left) and computed saliency maps (right). Top row has exudates and bottom row has drusen. Arrows point to Subtle lesions

Comparison with Other Detection Methods- Several methods for bright lesion detection can be found in literature. The SN/SP and (AUC) figures for some of the methods are as follows. For HE detection, [8] report 1/0.19 with AUC of 0.86 on HEI-MED dataset; [6] and [14] report $0.7 / 0.85$ and $0.7 / 0.99$ respectively on Diaretdb1, with [6] using a different set for training; [7] report an AUC of 0.96 on 400 images of MESSIDOR dataset. For drusen detection, [15] report $0.84 / 0.52$ on ARIA dataset. The performance of the proposed method (see table 2) is better than or comparable to all methods except [8] which trades off high SN for poor SP.

Most of the methods targeting bright lesion detection eventually report their classification performance for only HE or drusen at image level. An exception is [5] which follows a pixel-level detection of bright lesion candidates, clusters and labels them and finally distinguishes them as $\mathrm{HE}$ or drusen. A SN/SP=0.95/0.88 is reported on a custom dataset of size 300 images with both drusen and HE cases. The image level decision is based on detecting at least one

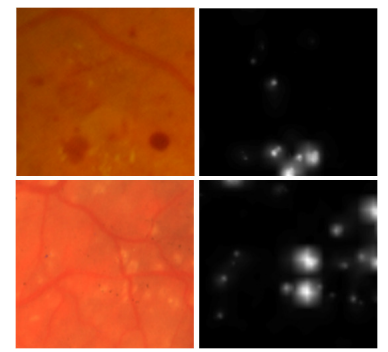

Fig. 3. Retinal image patches (first column) with low contrast exudates (first row) and drusen (second row) and their saliency maps (second column) 
Table 2. Performance of bright lesion detection

\begin{tabular}{lcccc}
\hline \hline Dataset & SN & SP & AUC & 5-fold AUC \\
\hline \hline Hard Exudates & & & & \\
\hline HEI-MED & 1 & 0.45 & 0.90 & 0.89 \\
\hline DiaretDB & 0.81 & 0.92 & 0.88 & 0.84 \\
\hline MESSIDOR & 0.91 & 0.82 & 0.93 & 0.89 \\
\hline \hline Drusen & & & & \\
\hline ARIA & 0.98 & 0.80 & 0.96 & 0.92 \\
\hline STARE & 0.98 & 0.82 & 0.98 & 0.95 \\
\hline \hline Combined & 0.91 & 0.76 & 0.91 & 0.89 \\
\hline \hline
\end{tabular}

HE or druse. In contrast, our saliency-based global approach achieves $0.91 / 0.76$ on 758 images (Combined dataset) drawn from 5 public datasets.

Discrimination of Bright Lesions (level 2)- A total of 388 abnormal images from the Combined dataset was considered for this study. Of these, there were 171 drusen cases and $217 \mathrm{HE}$ cases. This set was randomly divided into two equal parts for training and testing. The training and testing process was repeated 10 times, with a random selection of training and testing data each time. The average classification accuracy figures are reported in table 3 . The obtained high accuracy, even with a simple LBP feature, underscores the key role saliency plays in identifying $P_{L}$. Correct identification depends on $x$, with a low value potentially leading to extraction of irrelevant image patches and a high $x$ leading to omission of abnormal regions. This in turn affects discrimination as seen from Table 3 .

Table 3. Performance of discrimination between HE and drusen.

\begin{tabular}{ccc}
\hline \hline Threshold $(x \%)$ & Avg. no of patches & Accuracy $(\%)$ \\
\hline 20 & 3.08 & 96.41 \\
\hline 40 & 2.14 & 95.31 \\
\hline 80 & 1.19 & 93.24 \\
\hline \hline
\end{tabular}

\section{CONCLUSION}

In this paper, we explored if visual saliency can have a role in CAD algorithm design. We have proposed a novel, visual saliency based framework for abnormality detection and illustrated it for bright lesion detection and discrimination in retinal images. The proposed framework is evaluated on several public datasets. The obtained results indicate that saliency maps are a good source of information for detecting/discriminating bright lesions. There is much scope to explore as there are numerous available saliency models of which one was applied in this work.

Extending the proposed framework to more challenging retinal abnormalities such as spot lesions like microaneurysms (which are very subtle) would be interesting. The proposed framework may need to employ saliency computation models which permit inclusion of top-down information to handle such challenges.

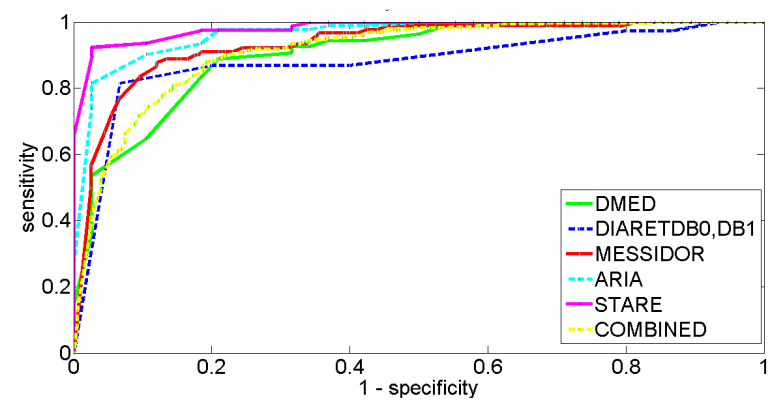

Fig. 4. ROC curve for bright lesion detection

\section{REFERENCES}

[1] Nodine et.al, "Using eye movements to study visual search and to improve tumor detection," Radiographics, vol. 7, no. 6, pp. 1241-1250, 1987.

[2] Matsumoto et.al, "Where do neurologists look when viewing brain ct images? an eye-tracking study involving stroke cases," PloS one, vol. 6, no. 12, pp. e28928, 2011.

[3] Harel et.al, "Graph-based visual saliency," in NIPS, 2006, pp. 545-552.

[4] Jampani et.al, "Assessment of computational visual attention models on medical images," in Indian conf. on vis. graphics and img. proc. ICVGIP, 2012.

[5] Niemeijer et.al., "Automated detection and differentiation of drusen, exudates, and cotton-wool spots in digital color fundus photographs for diabetic retinopathy diagnosis," Invest Ophthalmol Vis Sci., vol. 48, no. 5, pp. 2260-2267, 2007.

[6] Rocha et.al, "Points of interest and visual dictionaries for automatic retinal lesion detection," Biomed. Engg., IEEE Trans. on, vol. 59, no. 8, pp. $2244-2253$, aug. 2012.

[7] KS. Deepak and J. Sivaswamy, "Automatic assessment of macular edema from color retinal images," IEEE Trans. Med. Img., vol. 31, no. 3, pp. 766-776, 2012.

[8] Giancardo et.al., "Automatic retina exudates segmentation without a manually labelled training set," in ISBI, 30 2011-april 2 2011, pp. 1396 -1400 .

[9] Agurto et.al, "Multiscale am-fm methods for diabetic retinopathy lesion detection.," IEEE Trans. Med. Imaging, vol. 29, no. 2, pp. 502$512,2010$.

[10] Freund et.al, "Automated detection of drusen in the macula," 2009, ISBI'09, pp. 61-64.

[11] Quellec et.al, "Optimal filter framework for automated, instantaneous detection of lesions in retinal images.," IEEE Trans. Med. Imaging, vol. 30, pp. 523-533, 2011.

[12] X. Hou and L. Zhang, "Saliency detection: A spectral residual approach," in CVPR07, 2007, pp. 1-8.

[13] Deepak et.al, "Detection and discrimination of disease-related abnormalities based on learning normal cases," $P R$, vol. 45, no. 10, pp. 37073716, 2012.

[14] Welfer et.al., "A coarse-to-fine strategy for automatically detecting exudates in color eye fundus images," Comp. Med. Img. Graphics CMIG, vol. 34, no. 3, pp. $228-235,2010$.

[15] Hijazi et.al, "Retinal image classification for the screening of agerelated macular degeneration," in The 30th SGAI Int. Conf. on Innovative Tech. and App. of AI, 2010, pp. 325-338.

[16] L. Brandon and A. Hoover, "Drusen detection in a retinal image using multi-level analysis," in MICCAI, 2003, pp. 618-625. 